\title{
Die Bedeutung von Kreativität (nicht nur) im Recht
}

\author{
Braucht es gar ein Recht auf Kreativität?
}

\author{
C LA U D I A T T O
}

Nach einer Studie der University of Minnesota in 2013 fördert eine unordentliche Umgebung einen kreativen Geist. "Ungeordnete Umgebungen scheinen zu inspirieren, sich von Traditionen zu befreien, was zu neuen Erkenntrissen führen kann", schloss Studienleiterin Vohs aus den Ergebnissen der Studie. "Ordentliche Umgebungen hingegen fördern Konventionen und gehen auf Nummer sicher."2

Recht erfüllt nach Louven eine Ordnungsfunktion. ${ }^{3}$ Recht schafft (Rechts-)Sicherheit. Recht kann Kreativität also im Weg stehen. Wie Louven aufgezeigt hat, kann Recht Kreativität aber auch fordern oder fördern. Das zeigt sich nicht nur an komplexen Rechtsstreitigkeiten, die gelöst werden müssen. Sondern auch an neuen technologischen Sachverhalten, die das technologieneutrale Recht nicht explizit regelt. Hier kann Kreativität Erleichterung verschaffen.

Wer sich nicht als kreative Person versteht, findet in der Literatur zur Psychologie der Kreativität, v.a. im nachfolgenden Beitrag von Funke, ${ }^{4}$ hilfreiche Informationen und Tipps zur Förderung der eigenen Kreativität. Diese können auch kre- ativen Persönlichkeiten helfen, durch Routinen entstandene Betriebsblindheit zu überwinden.

Doch nicht nur der oder die Einzelne ist aufgerufen, kreatives Potential zu ermöglichen und zu entfalten. Schulen, Universitäten und andere Ausbildungsstätten sind die wohl wichtigsten Stellen, die Kreativität als wünschenswert vermitteln können. Auch die Unternehmen können die Kreativität der Mitarbeitenden fördern, die wiederum dem Unternehmen zugute kommen wird. Mit ein paar bunten Getränken zu bunten Sesseln in bunten Räumen ist es jedoch nicht getan. Innovation Hubs sind ein guter Ansatz, aber nicht immer gut umgesetzt. Vielmehr beginnt die Förderung von Kreativität bei der Offenheit gegenüber dem Infragestellen bestehender Strukturen und Verhaltensweisen. Also genau dort, wo grundsätzlich nichts geändert werden soll.

Kreativität braucht Raum: Im Kopf und im Umfeld. Kreativität kann nicht angeordnet, sondern muss eingeladen werden. Daher braucht es Offenheit für Änderung und die Akzeptanz auch von Unordnung, um Bedeutendes leisten zu können.

\footnotetext{
1 https://www.psychologicalscience.org/news/releases/tidy-desk-or-messy-desk-each-has-its-benefits.html (zuletzt abgerufen am 24. Juni 2021)

2 https://www.psychologicalscience.org/news/releases/tidy-desk-or-messy-desk-each-has-its-benefits.html (zuletzt abgerufen am 24. Juni 2021).

3 Louven, Ri 2021, 49 (50).

4 Funke, Ri 2021, $61 \mathrm{ff}$.
} 